\title{
Genrikh Nikolayevich Berestovsky. Eightieth Anniversary Greetings
}

DOI: $10.1134 / \mathrm{S} 1990747809020196$

Prominent scientist Professor Genrikh N. Berestovsky became octogenarian on February 2, 2009. He belongs to the cohort of pioneers and founders of the biophysical field shortly termed as "artificial membranes". This field was turbulently developing in past, and now one is consolidated into an acknowledged and fruitful branch of biophysics. Prof. G. Berestovsky and his co-workers fulfilled a variety of experimental and theoretical works to unveil physico-chemical features of liposomes and lipid bilayers, to characterize transport processes mediated by certain channel formers in these biological systems, to reconstruct natural ion channels in lipid bilayers, and to examine those in situ. These studies provided a deep insight into our knowledge on the structure and function of artificial and cellular membranes. The scientific findings of G.N. Berestovsky and his disciples and data of their colleagues were summarized and generalized by G.N. Berestovsky and his co-author Prof. V.G. Ivkov in two monographs that are well cited till now.

Over many years, Prof. G.Berestovsky serves in the Scientific Councils of Russian Academy of Sciences on Biophysics and Biological Membranes; he is also an active member of the Editorial Boards of the journals Biofizika and Biologicheskie Membrany.

Within the last decade, Prof. G. Berestovsky focused on physiology of plant cells. The somewhat paradoxical result obtained by him and his co-workers is that the plant cells are capable of generating action potentials. This discovery prompted their pioneer studies of electrical excitability of plant cells. Using physiological, biochemical, and mathematical methods in combination, Prof. G. Berestovsky and co-workers succeeded in identifying and characterizing key ion channels and intracellular events, which mediate electrical excitability of plant cells, and elaborated a detailed mathematical model of membrane and intracellular processes enabling these cells to generate action potentials.

Professor G. Berestovsky efficiently combines his research with teaching. He served as an adviser on three doctoral and twenty $\mathrm{PhD}$ dissertations. Many students and postgraduates have practiced in the Department of Membrane Biophysics headed by G.N. Berestovsky and learned from him not only modern biophysics but also high standards of science and intelligence.

The Editorial of Biologicheskie Membrany congratulates our member Genrikh Berestovsky and thanks him for the generous support of the journal and valuable contribution to the board activity. We cordially wish our colleague and dear friend to be of good health and to remain a magnetic and creative personality for a long time.
Yu. A. Chizmadzhev, S. S. Kolesnikov 